\title{
Biomedical Nanotechnology Related Grand Challenges and Perspectives
}

\author{
Ajeet Kaushik* \\ Division of Sciences, Art, \& Mathematics, Department of Natural Sciences, Florida Polytechnic University, Lakeland, FL, \\ United States
}

Keywords: nanotechnology, smart diagnostics, nanomedicine, nano-sensors, drug delivery nano-systems, nanotheranostic, advanced therapies

\section{INTRODUCTION}

Biomedical nanotechnology is dedicated to exploring nanoscience and nanotechnology for health wellness, with the ultimate goal of personalized health management, as shown in Figure 1. Reports released by health agencies have confirmed the significant role technologies play in disease monitoring, treatment, and progression management. It is also evident that the introduction of nanotechnology assisted approaches makes diagnostics and treatment of a targeted disease more sensitive, affordable, and accessible (Kaushik and Dixit, 2016; Kaushik et al., 2017c; Kaushik and Mujawar, 2018). The tunable performance of nano-systems, investigated for biomedical research, is advantageous for the design and development of therapies that consider patient profiles i.e., personalized health management (Nair et al., 2016; Kaushik et al., 2018a,b). Besides salient features of nano-assisted approaches, the introduction of numerical approaches i.e., artificial intelligence (AI) (Yu et al., 2018; Zhu and Zheng, 2018), involving deep \& machine learning, and bioinformatics

\section{OPEN ACCESS}

Edited by:

Bingqing Wei,

University of Delaware, United States

Reviewed by:

Won Min Park,

Kansas State University, United States

${ }^{*}$ Correspondence:

Ajeet Kaushik akaushik@floridapoly.edu

Specialty section:

This article was submitted to Biomedical Nanotechnology,

a section of the journal

Frontiers in Nanotechnology

Received: 14 October 2019 Accepted: 08 November 2019 Published: 28 November 2019

Citation:

Kaushik A (2019) Biomedical Nanotechnology Related Grand Challenges and Perspectives.

Front. Nanotechnol. 1:1.

doi: 10.3389/fnano.2019.00001
(Chou, 2004; Greene et al., 2014) has emerged as a very useful tool in understanding predictions and trends. Such information is useful to understand epidemic variations, therapy optimization, and risk assessment. At the same time, suitable management of bioinformatics is crucial for a timely and effective analysis (Altman and Levitt, 2018; Lesk, 2019). Presently, significant efforts are being made to promote the internet of medical things (IoMT), an approach in biomedical nanotechnology for data sharing, storage, and analysis (Chiuchisan et al., 2015; Yuehong et al., 2016; Rodrigues et al., 2018). IoMT outcomes are useful in transitioning biomedical technology from the laboratory to the field. Keeping these outcomes in mind, efforts are being made to transform electronic health to intelligent health (Eysenbach, 2001; Berrouiguet et al., 2018).

The significant contributions of state-the-of-art biomedical nanotechnologies along with its challenges are summarized below:

a) The foundation of biomedical nanotechnology is always a nano-scaled platform which exhibits tunable features such as surface functionalization (for immobilizing and binding bioactives), stimuli-responsive properties (controlled multi-functional outcomes within a single nanostructure, a need to a combinational approach), easy to fabricate (especially thin films needed for sensor fabrication), and tunable properties (especially morphological, optical, electrical, magnetic, and molecular properties). However, developing a nano-system that exhibits properties with targeted application in mind is always challenging (Figure 1; Kaushik and Dixit, 2016).

b) Detection of targeted biomarkers at $\mathrm{pM}$ level, is very useful for early-stage diagnostics and therapy efficacy assessment. However, significant efforts have been suggested to develop a miniaturized sensing system that is integrated with smartphones to perform the detection of point-of-care (POC) applications i.e., infectious diseases (Figure 1; Kaushik and Mujawar, 2018). 


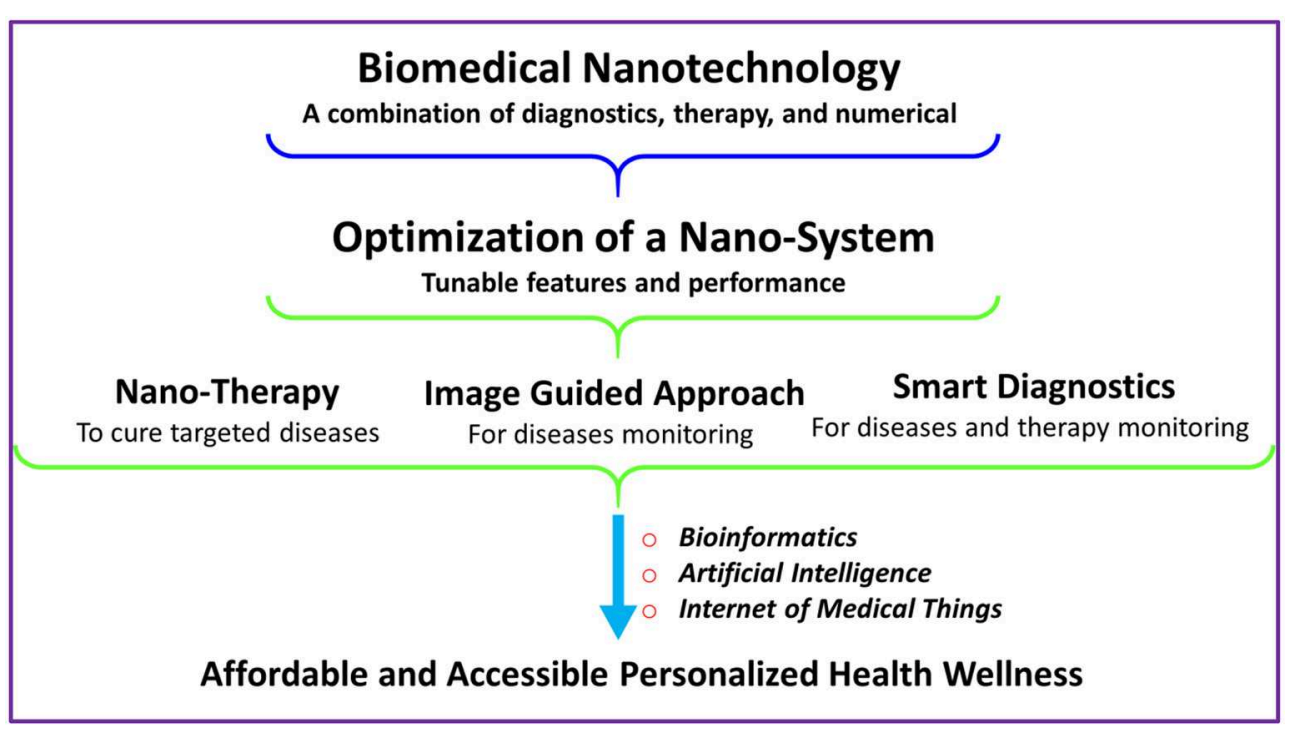

FIGURE 1 | Illustration of Biomedical Nanotechnology for personalized health care management.

c) Accurate and precise detection of a biomarker is the primary requirement for designing and developing an analytical device. This raises the demand for developing smart arrays (interdigitated electrode system), multiple detection methods, highly sensitive transducers, and microfluidic systems. A perfect combination of the above-mentioned components will certainly be a sensing system that can detect a biomarker selectively and in a timely manner, at a very low level of POC (Figure 1; Dixit and Kaushik, 2016; Kaushik and Dixit, 2016; Kaushik et al., 2016a,c,d, 2017b, 2018c; Tiwari et al., 2019).

d) Functional biomaterials for tissue engineering are mainly tissue regeneration and regenerative medicine. This area of research explores new bio-mimic biomaterials that are constructed to minimize the effect of tissue injury and artificial organ issues. However, exploring the fundamental understanding of biomaterial-organ interaction behavior and strategies of scaling up these biomaterials, along with federal approval for clinical application is the suggested future approach (Vashist et al., 2016, 2018b; Sharma et al., 2019).

e) Nanotechnology assisted drug delivery systems have demonstrated site-specific delivery and drug release to manage targeted diseases. Presently, this area of biomedical nanotechnology is widely covered, and outcomes are of very high value. However, so far, developed systems' multi component-based nano-assisted therapeutics, may have longer term side-effects. Experts have suggested the development of least-component based nano-pharmacology wherein selected drug nano-carriers should be biocompatible and stimuli-responsive. Such materials can perform controlled drug delivery and release, even in the brain (Figure 1; Kaushik et al., 2016b, 2019; Nair et al., 2016; Rodriguez et al., 2017; Vashist et al., 2018a). f) Nanotechnology assisted combinational therapy is emerging as one of the best alternatives in conventional approaches. The approach optimizes a perfect combination of various therapeutic agents, therapeutic fabrication processes, and stimulations to treat multiple symptoms, at one time, while exploring the effects of various stimulations, exposures, drug-to-drug interactions, and drug/stimulation-human interactions in acute and chronic timelines (Figure 1; Kaushik et al., 2017a; Tomitaka et al., 2017, 2019; Jayant et al., 2018).

g) The design and development of a new therapy is a wellplanned experiment. Managing a lot of data, optimization of a perfect drug to create a therapy, the associated risk assessment, and possible predictions along with trends are always major concerns. To manage data related to every aspect, a new algorithm has emerged that analyzes every aspect and provides an idea of a better approach with no or the least risk assessment. Keeping this in mind, the emergence of bioinformatics, AI, and IoMT significantly assist the development of novel biomedical nanotechnology (Figure 1; Chou, 2004; Greene et al., 2014; Chiuchisan et al., 2015; Yuehong et al., 2016; Altman and Levitt, 2018; Rodrigues et al., 2018; Yu et al., 2018; Zhu and Zheng, 2018; Lesk, 2019).

h) Health agencies like the World Health Organization (WHO), the National Institutes of Health (NIH), etc., have announced the development of biomedical technology with a special focus on efficient approaches that manage and target diseases. They have initiated various programs, alone and in collaboration with industries, to promote fundamental and applied aspects of biomedical nanotechnologies. These initiatives aim to develop translational research to introduce new therapies at the clinical level. However, poor regularities and the time required for approval sometimes limit the 
promotion of developed biomedical nanotechnology for patients. Significant efforts are being made to involve a multi-sector, mainly public-private partnerships, to promote research from a clinical translational point of view (Figure 1; Kaushik et al., 2018b).

i) Special assertion from the WHO, NIH, etc., type of health agencies is also required to make people aware of the significance of biomedical nanotechnology. For example, gene therapy has emerged as a potential therapeutic approach, but some people are not comfortable in adopting it as a therapy. Therefore, awareness is also really very important as with conducting cutting edge research (Figure 1; Kaushik et al., 2018b).

\section{BIOMEDICAL NANOTECHNOLOGY, A GREAT INITIATIVE OF FRONTIERS OF NANOTECHNOLOGY}

As discussed above, biomedical nanotechnology is needed for a better future. Therefore, highlighting the need for the promotion and related critical aspects of biomedical nanotechnology is very important for education and training. Keeping this in mind, the mission of the Biomedical Nanotechnology Journal featured in the Frontiers in Nanotechnology Journal will aim at exploring the fundamentals as well as applied research investigating novel nano-enabled therapeutics and diagnostic approaches to developing effective and affordable therapies and diagnostics. The outcomes of these approaches, alone or in combination, will certainly be a useful disease management approach that aims to improve personalized health needs. Based on these objectives, this Journal will cover all aspects of fundamental and advanced research related to the following areas:

- Nanoscale for biomedical applications

- Miniaturized systems for health care

- Nano-enabled sensing systems

- Simulation and theoretical aspects of developing sensors

- Point-of-care systems for personalized health care

- Microfluidic for biologist

- Image-guided therapy

- Personalized nanomedicine

- Nano-enabled tissues and gene engineering

- Nanotechnology for drug delivery systems

- Nano-pharmacology

- Nanobiotechnology for drug addiction

- Translational and clinical research

- Theoretical aspects of diseases managements

- Bioinformatics for diseases management

- Artificial Intelligence for biomedical application

- Internet of medical things (IoMT)

- Numerical aspects of nanotechnology for health care

- Ethical and regulatory issues in theranostics.

An editorial board of experts along with the advanced publication platform offered at Frontiers this Journal will be fully dedicated to publishing high-quality Original Research articles. In addition to high-quality Original Research articles, this Journal will also publish Technical Notes, Opinion articles, research highlights, brief communications, Letters, Book Reviews, comprehensive Reviews, and important announcements. This Journal will pay special attention to Published Book series related to all the fields of nano-enabling advancements in biomedical science.

This Journal will be a unique platform that provides an understanding of the aspects of smart nanomaterials, nano-devices for biomedical application, rapid diagnostics, and effective therapeutics developed using nanoscience and nanotechnology. The possibilities of numerical simulations, theoretical aspects, regulatory issues, and the ethical knowledge required to manage clinical and translational research of a targeted disease will be covered here. To support the aims of Frontiers, this Journal will serve as a guide to researchers in future research strategies on developing nano-enabled smart and effective diagnostics and therapies for health wellness.

\section{VIEWPOINT}

Despite the significant contribution of biomedical nanotechnology in health care management, significant efforts are being made to overcome the challenges of poor reproducibility, specificity and efficacy, affordability, and to avoid the regulatory landscape associated with state-the-ofart biomedical nanotechnology. Keeping advancements and prospects in mind, experts suggest increasing efforts to build new systems that meet patient requirements. Health agencies are seeking developments that can make health accessible, affordable, and manageable. This could be achieved by focusing efforts on the design and development of smart and effective nano-enabled components, which can elevate diagnostics and therapies to the desired level of performance. This Journal explores the demand, significance, challenges, and prospects of biomedical nanotechnology for personalized health wellness. We also encourage experts in the field to try their level best to solve and explore problems, by conducting cutting edge research in biomedical nanotechnology. To support the research of industries, institutions, and universities, the supporting and motivating policies of federal agencies are also very important. Overall, balanced research, supported by publicprivate partnerships is suggested to develop and promote the biomedical nanotechnology of health care management in a personalized manner.

\section{AUTHOR CONTRIBUTIONS}

The author confirms being the sole contributor of this work and has approved it for publication.

\section{ACKNOWLEDGMENTS}

I acknowledge Frontiers for providing the publication platform and opportunity. The facilities and resources of the Department of Natural Sciences, Division of Sciences, Art, \& Mathematics, Florida Polytechnic University, Lakeland, FL-33805, USA are also acknowledged in this report. 


\section{REFERENCES}

Altman, R. B., and Levitt, M. (2018). What is biomedical data science and do we need an annual review of it? Annu. Rev. Biomed. Data Sci. 1, 1-3. doi: 10.1146/annurev-bd-01-041718-100001

Berrouiguet, S., Perez-Rodriguez, M. M., Larsen, M., Baca-García, E., Courtet, P., and Oquendo, M. (2018). From eHealth to iHealth: transition to participatory and personalized medicine in mental health. J. Med. Intern. Res. 20:e2. doi: 10.2196/jmir.7412

Chiuchisan, I., Chiuchisan, I., and Dimian, M. (2015). "Internet of Things for e-Health: an approach to medical applications," in 2015 International Workshop on Computational Intelligence for Multimedia Understanding (IWCIM) (Prague: IEEE), 105. doi: 10.1109/IWCIM.2015.7347091

Chou, K. C. (2004). Structural bioinformatics and its impact to biomedical science. Curr. Med. Chem. 11, 2105-2134. doi: 10.2174/0929867043364667

Dixit, C. K., Kaushik, A. K. (2016). Microfluidics for Biologists. Berlin: Springer. doi: 10.1007/978-3-319-40036-5

Eysenbach, G. (2001). What is e-health? J. Med. Intern. Res. 3:e20. doi: 10.2196/jmir.3.2.e20

Greene, C. S., Tan, J., Ung, M., Moore, J. H., and Cheng, C. (2014). Big data bioinformatics. J. Cell. Physiol. 229, 1896-1900. doi: 10.1002/jcp.24662

Jayant, R. D., Tiwari, S., Atluri, V., Kaushik, A., Tomitaka, A., Yndart, A., et al. (2018). Multifunctional nanotherapeutics for the treatment of neuroAIDS in drug abusers. Sci. Rep. 8:12991. doi: 10.1038/s41598-018-31285-w

Kaushik, A., and Dixit, C. K. (eds.). (2016). Nanobiotechnology for Sensing Applications: From Lab to Field. New York, NY: CRC Press. doi: $10.1201 / 9781315366425$

Kaushik, A., Jayant, R. D., Bhardwaj, V., and Nair, M. (2018b). Personalized nanomedicine for CNS diseases. Drug Discov. Today 23, 1007-1015. doi: 10.1016/j.drudis.2017.11.010

Kaushik, A., Jayant, R. D., and Nair, M. (2018a). Nanomedicine for neuroHIV/AIDS management. Nanomedicine 13, 669-673. doi: 10.2217/nnm-2018-0005

Kaushik, A., Jayant, R. D., and Nair, M. (eds.). (2017c). Advances in Personalized Nanotherapeutics. Cham: Springer. doi: 10.1007/978-3-319-63633-7

Kaushik, A., Jayant, R. D., Nikkhah-Moshaie, R., Bhardwaj, V., Roy, U., Huang, Z., et al. (2016b). Magnetically guided central nervous system delivery and toxicity evaluation of magneto-electric nanocarriers. Sci. Rep. 6:25309. doi: 10.1038/srep25309

Kaushik, A., Jayant, R. D., Tiwari, S., Vashist, A., and Nair, M. (2016a). Nanobiosensors to detect beta-amyloid for Alzheimer's disease management. Biosens. Bioelectron. 80, 273-287. doi: 10.1016/j.bios.2016.01.065

Kaushik, A., and Mujawar, M. (2018). Point of care sensing devices: better care for everyone. Sensors 18, 4303. doi: 10.3390/s18124303

Kaushik, A., Nikkhah-Moshaie, R., Sinha, R., Bhardwaj, V., Atluri, V., Jayant, R. D., et al. (2017a). Investigation of ac-magnetic field stimulated nanoelectroporation of magneto-electric nano-drug-carrier inside CNS cells. Sci. Rep. 7:45663. doi: $10.1038 /$ srep45663

Kaushik, A., Tiwari, S., Jayant, R. D., Marty, A., and Nair, M. (2016c). Towards detection and diagnosis of Ebola virus disease at pointof-care. Biosens. Bioelectron. 75, 254-272. doi: 10.1016/j.bios.201 5.08.040

Kaushik, A., Tiwari, S., Jayant, R. D., Vashist, A., Nikkhah-Moshaie, R., El-Hage, N., et al. (2017b). Electrochemical biosensors for early stage Zika diagnostics. Trends Biotechnol. 35, 308-317. doi: 10.1016/j.tibtech.201 6.10 .001

Kaushik, A., Vabbina, P. K., Atluri, V., Shah, P., Vashist, A., Jayant, R. D., et al. (2016d). Electrochemical monitoring-on-chip (E-MoC) of HIV-infection in presence of cocaine and therapeutics. Biosens. Bioelectron. 86, 426-431. doi: 10.1016/j.bios.2016.06.086
Kaushik, A., Yndart, A., Atluri, V., Tiwari, S., Tomitaka, A., Gupta, P., et al. (2019). Magnetically guided non-invasive CRISPR-Cas9/gRNA delivery across blood-brain barrier to eradicate latent HIV-1 infection. Sci. Rep. 9:3928. doi: 10.1038/s41598-019-40222-4

Kaushik, A., Yndart, A., Kumar, S., Jayant, R. D., Vashist, A., Brown, A. N., et al. (2018c). A sensitive electrochemical immunosensor for label-free detection of Zika-virus protein. Sci. Rep. 8:9700. doi: 10.1038/s41598-018-28035-3

Lesk, A. (2019). Introduction to Bioinformatics. New York, NY: Oxford University Press.

Nair, M., Jayant, R. D., Kaushik, A., and Sagar, V. (2016). Getting into the brain: potential of nanotechnology in the management of NeuroAIDS. Adv. Drug Deliv. Rev. 103, 202-217. doi: 10.1016/j.addr.2016.02.008

Rodrigues, J. J., Segundo, D. B. D. R., Junqueira, H. A., Sabino, M. H., Prince, R. M., Al-Muhtadi, J., et al. (2018). Enabling technologies for the internet of health things. IEEE Access 6, 13129-13141. doi: 10.1109/ACCESS.2017.2789329

Rodriguez, M., Lapierre, J., Ojha, C. R., Kaushik, A., Batrakova, E., Kashanchi, F., et al. (2017). Intranasal drug delivery of small interfering RNA targeting Beclin1 encapsulated with polyethylenimine (PEI) in mouse brain to achieve HIV attenuation. Sci. Rep. 7:1862. doi: 10.1038/s41598-017-01819-9

Sharma, K., Mujawar, M. A., and Kaushik, A. (2019). State-of Art functional biomaterials for tissue engineering. Front. Mater. 6:172. doi: $10.3389 /$ fmats. 2019.00172

Tiwari, S., Sharma, V., Mujawar, M., Mishra, Y. K., Kaushik, A., and Ghosal, A. (2019). Biosensors for epilepsy management: state-of-art and future aspects. Sensors 19:1525. doi: 10.3390/s19071525

Tomitaka, A., Arami, H., Raymond, A., Yndart, A., Kaushik, A., Jayant, R. D., et al. (2017). Development of magneto-plasmonic nanoparticles for multimodal image-guided therapy to the brain. Nanoscale 9, 764-773. doi: 10.1039/C6NR07520G

Tomitaka, A., Kaushik, A., Kevadiya, B., Mukadam, I., Gendelman, H. E., Khalili, K., et al. (2019). Surface-engineered multimodal magnetic nanoparticles to manage CNS diseases. Drug Discov. Today 24, 873-882. doi: 10.1016/j.drudis.2019.01.006

Vashist, A., Kaushik, A., Vashist, A., Bala, J., Nikkhah-Moshaie, R., Sagar, V., et al. (2018a). Nanogels as potential drug nanocarriers for CNS drug delivery. Drug Discov. Today 23, 1436-1443. doi: 10.1016/j.drudis.2018.05.018

Vashist, A., Kaushik, A., Vashist, A., Jayant, R. D., Tomitaka, A., Ahmad, S., et al. (2016). Recent trends on hydrogel based drug delivery systems for infectious diseases. Biomater. Sci. 4, 1535-1553. doi: 10.1039/C6BM00276E

Vashist, A., Kaushik, A., Vashist, A., Sagar, V., Ghosal, A., Gupta, Y. K., et al. (2018b). Advances in carbon nanotubes-hydrogel hybrids in nanomedicine for therapeutics. Adv. Healthc. Mater. 7:1701213. doi: 10.1002/adhm.201701213

Yu, K. H., Beam, A. L., and Kohane, I. S. (2018). Artificial intelligence in healthcare. Nat. Biomed. Eng. 2:719. doi: 10.1038/s41551-018-0305-Z

Yuehong, Y. I. N., Zeng, Y., Chen, X., and Fan, Y. (2016). The internet of things in healthcare: an overview. J. Indus. Inform. Integr. 1, 3-13. doi: 10.1016/j.jii.2016.03.004

Zhu, L., and Zheng, W. J. (2018). Informatics, data science, and artificial intelligence. JAMA 320, 1103-1104. doi: 10.1001/jama.2018.8211

Conflict of Interest: The author declares that the research was conducted in the absence of any commercial or financial relationships that could be construed as a potential conflict of interest.

Copyright (c) 2019 Kaushik. This is an open-access article distributed under the terms of the Creative Commons Attribution License (CC BY). The use, distribution or reproduction in other forums is permitted, provided the original author $(s)$ and the copyright owner(s) are credited and that the original publication in this journal is cited, in accordance with accepted academic practice. No use, distribution or reproduction is permitted which does not comply with these terms. 